UDK 517.9

\author{
M. E. Dudkin, O. Yu. Dyuzhenkova
}

\title{
SINGULARLY PERTURBED RANK ONE LINEAR OPERATORS
}

\begin{abstract}
M. E. Dudkin, O. Y. Dyuzhenkova. Singularly perturbed rank one linear operators, Mat. Stud. 56 (2021), 162-175.

The basic principles of the theory of singularly perturbed self-adjoint operators are generalized to the case of closed linear operators with non-symmetric perturbation of rank one. Namely, firstly linear closed operators are considered that coincide with each other on a dense set in a Hilbert space. The theory of singularly perturbed self-adjoint operators arose from the need to consider differential expressions in such terms as the Dirac $\delta$-function. Since it is important to consider expressions given not only by symmetric operators, the generalization (transfer) of the basic principles of the theory of singularly perturbed self-adjoint operators in the case of non-symmetric ones is important problem. The main facts of the theory include the definition of a singularly perturbed linear operator and the resolvent formula in the cases of $\mathcal{H}_{-1}$-class and $\mathcal{H}_{-2}$-class. The paper additionally describes the possibility of the appearance a point of the point spectrum and the construction of a perturbation with a predetermined point. In comparison with self-adjoint perturbations, the description of perturbations by non-symmetric terms is unexpected. Namely, in some cases, when the perturbed by a vectors from $\mathcal{H}_{-2}$ operator can be conveniently described by methods of class $\mathcal{H}_{-1}$, that is impossible in the case of symmetric perturbations of a self-adjoint operator. The perturbation of self-adjoint operators in a nonsymmetric manner fully fits into the proposed studies. Such operators, for example, generalize models with nonlocal interactions, perturbations of the harmonic oscillator by the $\delta$-potentials, and can be used to study perturbations generated by a delay or an anticipation.
\end{abstract}

1. Introduction. The theory of singularly perturbed self-adjoint operators has several thousands of large and small works, collected in the monographs [1,3]. The omitted methods in $[1,3]$ are presented in [10]. In general, this theory arose from the need to consider differential expressions in such terms as the Dirac $\delta$-function. Since it is important to consider expressions given not only by symmetric operators, the generalization (transfer) of the basic principles of the theory of singularly perturbed self-adjoint operators in the case of non-symmetric ones is important problem. The main facts of the theory include the definition of a singularly perturbed linear operator and the resolvent formula in the cases of $\mathcal{H}_{-1}$-class and $\mathcal{H}_{-2}$-class. The paper additionally describes the possibility of the appearance a point of the point spectrum and the construction of a perturbation with a predetermined point. In comparison with self-adjoint perturbations, the description of perturbations by non-symmetric terms is unexpected. Namely, in some cases, when the perturbed by a vectors from $\mathcal{H}_{-2}$ operator can be conveniently described by methods of class $\mathcal{H}_{-1}$, that is impossible in the case of symmetric perturbations of a self-adjoint operator. Some aspects of

2010 Mathematics Subject Classification: 34A37, 47A10, 47A55, 47A75.

Keywords: singularly perturbed operator; scale of Hilbert spaces; non-symmetric perturbations; eigenvalue; eigenvector; Krein's formula.

doi:10.30970/ms.56.2.162-175

(C) M. E. Dudkin, O. Yu. Dyuzhenkova, 2021 
non-symmetric rank one perturbations of self-adjoint operators are considered in $[7,8]$. There are also some technical details of proofs, which we do not repeat here. The perturbation of self-adjoint operators in a non-symmetric manner fully fits into the proposed studies. Such operators, for example, generalize models with nonlocal interactions [2,4], perturbations of the harmonic oscillator by the $\delta$-potentials [11], and can be used to study perturbations generated by a delay or an anticipation.

2. Perturbations $\mathcal{H}_{-1}$-class. Let us consider a linear unbounded closed operator $A$ on the dense domain $\mathfrak{D}(A)$ in the separable Hilbert $\mathcal{H}$ space with the scalar product $(\cdot, \cdot)$ and the norm $\|\cdot\|=\sqrt{(\cdot, \cdot)}$. (The unboundedness condition of the operator is obligatory and without it our consideration is not meaningful.) Suppose, the set of regular points $\rho(A)$ of the operator $A$ is not empty. It is an essential condition for all statements of the article.

Using the operator $A$, we construct $A$-scale of Hilbert spaces [6]. Next, consider only part of it, namely the chain:

$$
\mathcal{H}_{-2} \supset \mathcal{H}_{-1} \supset \mathcal{H} \equiv \mathcal{H}_{0} \supset \mathcal{H}_{+1} \supset \mathcal{H}_{+2}
$$

where $\mathcal{H}_{+k}$ is the positive space with the norm $\|\varphi\|_{+k}=\left\|\left(\sqrt{A^{*} A}+I\right)^{k / 2} \varphi\right\|, \varphi \in \mathfrak{D}\left(\sqrt{A^{*} A}\right)$, $\mathcal{H}_{-k}$ is the completion of $\mathcal{H}$ with respect to the norm $\|f\|_{-k}=\left\|\left(\sqrt{A^{*} A}+I\right)^{-k / 2} f\right\|, f \in \mathcal{H}$, $k=1,2, A^{*}$ is adjoint to $A$ operator in $\mathcal{H}, I$ stands for the identity operator. Denote by $\langle\cdot, \cdot\rangle$ the dual scalar product for the couple of spaces $\mathcal{H}_{+k}$ and $\mathcal{H}_{-k}, k=1,2$.

The extension of the operator $A$ by continuity on $\mathcal{H}_{-k}$ we can consider as the bounded operator acting from $\mathcal{H}_{+k}$ into $\mathcal{H}_{-k}$ and as an unbounded operator in $\mathcal{H}_{-k}$ with the domain $\mathcal{H}_{+k}, k \in\{1,2\}$. Such an extension we define by $\mathbf{A}$.

Let us consider the linear operator $V=\alpha\left\langle\cdot, \omega_{1}\right\rangle \omega_{2}, \omega_{1}, \omega_{2} \in \mathcal{H}_{-k}, \alpha \in \mathbb{C} \backslash\{0\}, k=1,2$, in the chain (1).

The perturbed operator $\tilde{A}$ in $\mathcal{H}$ we understand as the restriction of the sum of bounded operators from $\mathcal{H}_{-k}$ onto $\mathcal{H}$ :

$$
\tilde{A}:=\left(\mathbf{A}+\alpha\left\langle\cdot, \omega_{1}\right\rangle \omega_{2}\right)\lceil\mathcal{H} .
$$

Denote by $\mathbf{A}^{*}$ the adjoint to $\mathbf{A}$ operator according to (1) [5].

The restriction procedure is not always convenient to use, so we propose the following definition.

Definition 1. Let $A$ be a linear unbounded closed operator in the separable Hilbert space $\mathcal{H}$. For $\omega_{1}, \omega_{2} \in \mathcal{H}_{-1} \backslash \mathcal{H}$, we put $\eta_{1}(\bar{z})=\left(\mathbf{A}^{*}-\bar{z}\right)^{-1} \omega_{1}, \eta_{2}(z)=(\mathbf{A}-z)^{-1} \omega_{2}, z \in \rho(\mathbf{A})$. The operator $\tilde{A} \in \mathcal{P}_{-1}^{1}(A)$ is called singularly rank one perturbed of $\mathcal{H}_{-1}$-class with respect to $A$, if it has the domain (for a fixed $z$ )

$$
\mathfrak{D}(\tilde{A})=\left\{\vartheta=\phi-\mathbf{b}_{z} \eta_{2}(z) \mid \phi \in \mathfrak{D}(A), \mathbf{b}_{z}=\frac{\left((A-z) \phi, \eta_{1}(\bar{z})\right)}{1 / \alpha+\left\langle(\mathbf{A}-z)^{-1} \omega_{2}, \omega_{1}\right\rangle}\right\},
$$

in the case $\left\langle(\mathbf{A}-z)^{-1} \omega_{2}, \omega_{1}\right\rangle \neq-1 / \alpha$; and

$$
\mathfrak{D}(\tilde{A})=\mathfrak{D}_{\mathcal{H}_{1}} \dot{+}\left\{c \eta_{2}(z)\right\}, \quad \mathfrak{D}_{\mathcal{H}_{1}}=\left\{\phi \in \mathfrak{D}(A):\left((A-z) \phi, \eta_{1}(\bar{z})\right)=0\right\}, c \in \mathbb{C},
$$

in the case $\left\langle(\mathbf{A}-z)^{-1} \omega_{2}, \omega_{1}\right\rangle=-1 / \alpha$; the action is defined by the rule:

$$
(\tilde{A}-z) \vartheta=(A-z) \phi .
$$


Remark 1. The action of the defined in (2) perturbed operator satisfies (5) on vectors (3), (4).

Proof of Remark 1. This fact is verified by direct substitution (3), (4) in (2). Such an substitution is possible only for $\mathcal{H}_{-1}$-class perturbations. Indeed, one has

$$
\begin{gathered}
(\tilde{A}-z) \vartheta=\left((\mathbf{A}-z)+\alpha\left\langle\cdot, \omega_{1}\right\rangle \omega_{2}\right) \vartheta=(\mathbf{A}-z)\left[\phi-\frac{\left((A-z) \phi, \eta_{1}(\bar{z})\right)}{1 / \alpha+\left\langle(\mathbf{A}-z)^{-1} \omega_{2}, \omega_{1}\right\rangle}\right] \eta_{2}(z)+ \\
+\alpha\left\langle\left[\phi-\frac{\left((A-z) \phi, \eta_{1}(\bar{z})\right)}{1 / \alpha+\left\langle(\mathbf{A}-z)^{-1} \omega_{2}, \omega_{1}\right\rangle}\right], \omega_{1}\right\rangle \omega_{2}=(A-z) \phi-\frac{\left((A-z) \phi, \eta_{1}(\bar{z})\right)}{1 / \alpha+\left\langle(\mathbf{A}-z)^{-1} \omega_{2}, \omega_{1}\right\rangle} \omega_{2}+ \\
+\alpha\left\langle\phi, \omega_{1}\right\rangle \omega_{2}-\alpha \frac{\left((A-z) \phi, \eta_{1}(\bar{z})\right)}{1 / \alpha+\left\langle(\mathbf{A}-z)^{-1} \omega_{2}, \omega_{1}\right\rangle}\left\langle\eta_{2}(z), \omega_{1}\right\rangle \omega_{2}= \\
=(A-z) \phi-\frac{\left((A-z) \phi, \eta_{1}(\bar{z})\right)}{1 / \alpha+\left\langle(\mathbf{A}-z)^{-1} \omega_{2}, \omega_{1}\right\rangle} \omega_{2}+ \\
+\alpha \frac{\left\langle\phi, \omega_{1}\right\rangle\left(1 / \alpha+\left\langle(\mathbf{A}-z)^{-1} \omega_{2}, \omega_{1}\right\rangle\right)-\left((A-z) \phi, \eta_{1}(\bar{z})\right)\left\langle\eta_{2}(z), \omega_{1}\right\rangle}{1 / \alpha+\left\langle(\mathbf{A}-z)^{-1} \omega_{2}, \omega_{1}\right\rangle} \omega_{2}= \\
=(A-z) \phi-\frac{\left((A-z) \phi, \eta_{1}(\bar{z})\right)}{1 / \alpha+\left\langle(\mathbf{A}-z)^{-1} \omega_{2}, \omega_{1}\right\rangle} \omega_{2}+ \\
+\alpha \frac{1 / \alpha\left\langle\phi, \omega_{1}\right\rangle+\left\langle\phi, \omega_{1}\right\rangle\left\langle(\mathbf{A}-z)^{-1} \omega_{2}, \omega_{1}\right\rangle-\left((A-z) \phi, \eta_{1}(\bar{z})\right)\left\langle\eta_{2}(z), \omega_{1}\right\rangle}{1 / \alpha+\left\langle(\mathbf{A}-z)^{-1} \omega_{2}, \omega_{1}\right\rangle} \omega_{2}= \\
(A-z) \phi-\frac{\left\langle(A-z) \phi, \eta_{1}(\bar{z})\right)}{1 / \alpha+\left\langle(\mathbf{A}-z)^{-1} \omega_{2}, \omega_{1}\right\rangle} \omega_{2}+\frac{\langle\alpha}{1 / \alpha+\left\langle(\mathbf{A}-z)^{-1} \omega_{2}, \omega_{1}\right\rangle} \omega_{2}=(A-z) \phi
\end{gathered}
$$

since $\left((\mathbf{A}-z) \phi, \eta_{1}(\bar{z})\right)=\left(\phi,\left(\mathbf{A}^{*}-\bar{z}\right) \eta_{1}(\bar{z})\right)=\left\langle\phi, \omega_{1}\right\rangle$, and $\left\langle(\mathbf{A}-z)^{-1} \omega_{2}, \omega_{1}\right\rangle=\left(\eta_{2}(z), \omega_{1}\right)$.

Remark 2. Let us explain that two cases are caused for the domain $\mathfrak{D}(\tilde{A})$ in $(3)$ and (4), in Definition 1, since the fixed point $z$ may belong to the point spectrum of the perturbed operator, namely $z \notin \sigma_{p}(\tilde{A})$, as provided in the case $(3)$, and may belong to $\sigma_{p}(\tilde{A})$, namely $z \in \sigma_{p}(\tilde{A})$, as provided in the case $(4)$.

Remark 3. If we put $A$ as a self-adjoint operator, $\omega_{1}=\omega_{2}, \alpha \in \mathbb{R} \backslash\{0\}$, in Definition 1 then, obviously, we obtain known definition of the singularly perturbed self-adjoint operator of $\mathcal{H}_{-1}$-class $[3,10]$.

Denote a resolvent $R_{z}=(A-z)^{-1}, z \in \rho(A)$ of unperturbed operator $A$ and write the general form of the resolvent $\tilde{R}_{z}=(\tilde{A}-z)^{-1}, z \in \rho(\tilde{A})$ of perturbed operator $\tilde{A}$ in $\mathcal{H}$.

Theorem 1. Let $A$ be linear unbounded closed operator in the separable Hilbert space $\mathcal{H}$ and $\tilde{A} \in \mathcal{P}_{-1}^{1}(A)$ is a singularly non-symmetrically perturbed rank one with respect to $A$ operator, defined by (3)-(5). Then, the resolvent $R_{z}$ and resolvent $\tilde{R}_{z}$ are related to each other by M. Krein's type formula:

$$
\tilde{R}_{z}=R_{z}+b_{z}\left(\cdot, \eta_{1}(\bar{z})\right) \eta_{2}(z)
$$

with vector-valued functions

$$
\eta_{1}(\bar{z})=\left(A^{*}-\bar{\xi}\right)\left(A^{*}-\bar{z}\right)^{-1} \eta_{1}(\bar{\xi}), \eta_{2}(z)=(A-\xi)(A-z)^{-1} \eta_{2}(\xi)
$$


where $\eta_{1}(\bar{z}), \eta_{2}(z) \in \mathcal{H}_{+1}, z, \xi \in \rho(A) \cap \rho(\tilde{A})$, and the scalar-valued function

$$
-b_{z}^{-1}=\alpha^{-1}+\left\langle(\mathbf{A}-z)^{-1} \omega_{2}, \omega_{1}\right\rangle, \quad 0<|\alpha|<\infty,
$$

satisfies the condition

$$
b_{z}^{-1}-b_{\xi}^{-1}=(\xi-z)\left(\eta_{2}(\xi), \eta_{1}(\bar{z})\right),
$$

where the vectors $\eta_{1}(\bar{z}), \eta_{2}(z)$ are related to the vectors $\omega_{1}, \omega_{2}$ by the expression

$$
\eta_{1}(\bar{z})=\left(\mathbf{A}^{*}-\bar{z}\right)^{-1} \omega_{1}, \quad \eta_{2}(z)=(\mathbf{A}-z)^{-1} \omega_{2} .
$$

We can also consider the case $\alpha=0$, if we put $b_{z} \equiv 0$ and $\tilde{R}_{z} \equiv R_{z}$. There we can also consider $|\alpha|=\infty$. In such a case the term $\alpha^{-1}$ will omit in (8). In the theory of singularly perturbed self-adjoint operators the condition $|\alpha|=\infty$ corresponds to the case of the Friedrichs extension of a symmetric operator which is the joint for $A=A^{*}$ and $\tilde{A}=\tilde{A}^{*}$.

Proof. Let us consider the expression $\tilde{A}=A+\alpha\left\langle\cdot, \omega_{1}\right\rangle \omega_{2}$ in $\mathcal{H}_{-1}$, where $0<|\alpha|<\infty$. Remind that we use the notation $A$ i $\tilde{A}$ instead of $\mathbf{A}$ and $\tilde{\mathbf{A}}$. Then for some $z \in \rho(A)$ such that $\left((A-z)^{-1} \omega_{2}, \omega_{1}\right) \neq-\alpha^{-1}$, we write $(\tilde{A}-z)=(A-z)+\alpha\left\langle\cdot, \omega_{1}\right\rangle \omega_{2}$, and hence

$$
(\tilde{A}-z)^{-1}=(A-z)^{-1}-\alpha\left\langle\cdot,\left(A^{*}-\bar{z}\right)^{-1} \omega_{1}\right\rangle(\tilde{A}-z)^{-1} \omega_{2} .
$$

For the vector $\omega_{2}$ we have

$$
\begin{gathered}
(\tilde{A}-z)^{-1} \omega_{2}=(A-z)^{-1} \omega_{2}-\alpha\left\langle\omega_{2},\left(A^{*}-\bar{z}\right)^{-1} \omega_{1}\right\rangle(\tilde{A}-z)^{-1} \omega_{2}, \\
(\tilde{A}-z)^{-1} \omega_{2}=\left[1+\alpha\left\langle\omega_{2},\left(A^{*}-\bar{z}\right)^{-1} \omega_{1}\right\rangle\right](\tilde{A}-z)^{-1} \omega_{2}, \\
(\tilde{A}-z)^{-1} \omega_{2}=\frac{\alpha}{\left.1+\alpha\left\langle\omega_{2},\left(A^{*}-\bar{z}\right)^{-1} \omega_{1}\right\rangle\right\}}(A-z)^{-1} \omega_{2} .
\end{gathered}
$$

Substitute the last expression in (11), we get

$$
(\tilde{A}-z)^{-1}=(A-z)^{-1}-\frac{1}{\left.1 / \alpha+\left\langle\omega_{2},\left(A^{*}-\bar{z}\right)^{-1} \omega_{1}\right\rangle\right\}}\left(\cdot,\left(A^{*}-\bar{z}\right)^{-1} \omega_{1}\right)(A-z)^{-1} \omega_{2} .
$$

And now, there is need to redefine

$$
\begin{gathered}
\eta_{1}(\bar{z})=(A-\bar{z})^{-1} \omega_{1}, \eta_{2}(z)=(A-z)^{-1} \omega_{2}, \\
\left\langle\omega_{2},\left(A^{*}-\bar{z}\right)^{-1} \omega_{1}\right\rangle=\left\langle(A-z)^{-1} \omega_{2}, \omega_{1}\right\rangle=\left\langle(A-z) \eta_{2}(z), \eta_{1}(\bar{z})\right\rangle .
\end{gathered}
$$

From (10) we have $\eta_{1}(z)=R_{z} \omega_{1}, \eta_{1}(\xi)=R_{\xi} \omega_{1}$ and $\omega_{1}=(A-\xi) \eta_{1}(\xi)$, i.e.

$$
\eta_{1}(z)=R_{z}(A-\xi) \eta_{1}(\xi) .
$$

The last expression is correct only for the space $\mathcal{H}_{-1}$. For the correctness in $\mathcal{H}$ we must write in the form $\eta_{1}(z)=(A-\xi) R_{z} \eta_{1}(\xi)$, since $(A-\xi)$ commutes with $R_{z}$ and $\eta_{1}(z), \eta_{1}(\xi) \in$ $\mathcal{H}_{+1} \subset \mathcal{H}$.

Analogously, expressions $\eta_{2}(z)=R_{z} \omega_{2}$ and $\eta_{2}(\xi)=R_{\xi} \omega_{2}$ give $\eta_{2}(z)=(A-\xi) R_{z} \eta_{2}(\xi)$.

Hence, (7) is proved. 
Let us prove (9). Using (8), we have

$$
\begin{gathered}
b_{z}^{-1}-b_{\xi}^{-1}=-\left\langle\omega_{2},\left(A^{*}-\bar{z}\right)^{-1} \omega_{1}\right\rangle+\left\langle\omega_{2},\left(A^{*}-\bar{\xi}\right)^{-1} \omega_{1}\right\rangle= \\
=\left\langle\omega_{2},\left(\left(A^{*}-\bar{\xi}\right)^{-1}-\left(A^{*}-\bar{z}\right)^{-1}\right) \omega_{1}\right\rangle=\left\langle\left((A-\xi)^{-1}-(A-z)^{-1}\right) \omega_{2}, \omega_{1}\right\rangle= \\
=\left\langle(\xi-z)(A-\xi)^{-1}(A-z)^{-1} \omega_{2}, \omega_{1}\right\rangle= \\
=(\xi-z)\left\langle(A-\xi)^{-1} \omega_{2},(A-\bar{z})^{-1} \omega_{1}\right\rangle=(\xi-z)\left(\eta_{2}(\xi), \eta_{1}(\bar{z})\right) .
\end{gathered}
$$

And conversely, for some $h \in \mathcal{H},(\tilde{A}-z)^{-1} h=\vartheta \in \mathfrak{D}(\tilde{A})$. Hence, we take $(A-z)^{-1} h=\phi$ and substitute it in (6):

$$
\begin{gathered}
\vartheta=(\tilde{A}-z)^{-1} h=(A-z)^{-1} h+b_{z}\left(h, \eta_{1}(\bar{z})\right) \eta_{2}(z)= \\
=\phi-\frac{1}{1 / \alpha+\left\langle(A-z) \eta_{2}(z), \eta_{1}(\bar{z})\right\rangle}\left(h, \eta_{1}(\bar{z})\right) \eta_{2}(z)=\phi-\frac{\left((A-z) \phi, \eta_{1}(\bar{z})\right)}{1 / \alpha+\left\langle(A-z) \eta_{2}(z), \eta_{1}(\bar{z})\right\rangle} \eta_{2}(z) .
\end{gathered}
$$

Comparing the start and the end of the last formula, we obtain (3).

Theorem 1 gives directly an important consequence.

Corollary 1. The domain $\mathfrak{D}(\tilde{A})$ of the operator $\tilde{A} \in \mathcal{P}_{-1}^{1}(A)$ does not depend on the choice of the point $z \in \rho(A)$ in Definition 1.

Proof. Let us plug a vector $(A-z) \phi$ into $(6)$. We obtain $\vartheta=\phi+b_{z}\left((A-z) \phi, \eta_{1}(\bar{z})\right) \eta_{2}(z)$, i.e. it is the vector of $(3)$ for a fixed $z$. Substituting $(A-\xi) \phi$ into $(6)$ for $\xi$, we also obtain

$$
\vartheta=\phi+b_{z}\left((A-\xi) \phi, \eta_{1}(\bar{\xi})\right) \eta_{2}(\xi) \in \mathfrak{D}(\tilde{A}) .
$$

The second way to show the independence $\mathfrak{D}(\tilde{A})$ of $z$ is direct verification of the equality

$$
b_{z}\left((A-z) \phi, \eta_{1}(\bar{z})\right) \eta_{2}(z)=b_{z}\left((A-\xi) \phi, \eta_{1}(\bar{\xi})\right) \eta_{2}(\xi)
$$

using (7), (9), and the Hilbert identity. Then we rewrite mean calculations of the proof of Theorem 1.

By analogy to $(\tilde{A}-z)$ we define $\left((\tilde{A})^{*}-\bar{z}\right)$ for a fixed $z$.

$$
\mathfrak{D}\left((\tilde{A})^{*}\right)=\left\{\vartheta=\phi-\mathbf{b}_{z} \eta_{1}(\bar{z}): \phi \in \mathfrak{D}\left(A^{*}\right), \mathbf{b}_{z}=\frac{\left(\left(A^{*}-\bar{z}\right) \phi, \eta_{2}(z)\right)}{1 / \bar{\alpha}+\left\langle\left(\mathbf{A}^{*}-\bar{z}\right)^{-1} \omega_{1}, \omega_{2}\right\rangle}\right\}
$$

in the case $\left(\left(\mathbf{A}^{*}-\bar{z}\right)^{-1} \omega_{1}, \omega_{2}\right) \neq-1 / \bar{\alpha}$; and

$$
\mathfrak{D}\left((\tilde{A})^{*}\right)=\mathfrak{D}_{\mathcal{H}_{1}}^{*} \dot{+}\left\{c \eta_{1}(\bar{z})\right\}, \mathfrak{D}_{\mathcal{H}_{1}}^{*}=\left\{\phi \in \mathfrak{D}\left(A^{*}\right):\left(\left(A^{*}-\bar{z}\right) \phi, \eta_{2}(z)\right)=0\right\}
$$

in the case $\left((\mathbf{A}-\bar{z})^{-1} \omega_{1}, \omega_{2}\right)=-1 / \bar{\alpha}$; the action is defined analogously to $(5)$ :

$$
\left((\tilde{A})^{*}-\bar{z}\right) \vartheta=\left(A^{*}-\bar{z}\right) \phi .
$$

Now another corollary of Theorem 1 is obvious.

Corollary 2. If the operator $A$ (and its adjoint $A^{*}$ ) are given, then we have the equality $(\tilde{A}-z)^{*}=\tilde{A}^{*}-\bar{z}, z \in \rho(A)$ for the operator $\tilde{A}-z$ defined in (3)-(5) and $\tilde{A}^{*}-\bar{z}$ defined in in (12)-(14). 
Proof. Indeed, the expression adjoint to (6) has the form

$$
\left(\tilde{R}_{z}\right)^{*}=\left(R_{z}+b_{z}\left(\cdot, \eta_{1}(\bar{z})\right) \eta_{2}(z)\right)^{*}=R_{z}^{*}+b_{z}^{*}\left(\cdot, \eta_{2}(z)\right) \eta_{1}(\bar{z})=R_{z}^{*}+b_{\bar{z}}\left(\cdot, \eta_{2}(z)\right) \eta_{1}(\bar{z}),
$$

where $b_{\bar{z}}=b_{z}^{*}=\frac{1}{1 / \bar{\alpha}+\left\langle\left(A^{*}-\bar{z}\right) \omega_{1}, \omega_{2}\right\rangle}$. From (8) we have

$$
\begin{aligned}
-\overline{b_{\bar{z}}} & =\overline{1 / \alpha+\left\langle(A-z)^{-1} \omega_{2}, \omega_{1}\right\rangle}=1 / \bar{\alpha}+\overline{\left\langle(A-z)^{-1} \omega_{2}, \omega_{1}\right\rangle}= \\
& =1 / \bar{\alpha}+\left\langle\left(\omega_{1},(A-z)^{-1} \omega_{2}\right\rangle=1 / \bar{\alpha}+\left\langle\left(\left(A^{*}-\bar{z}\right)^{-1} \omega_{1}, \omega_{2}\right\rangle=-b_{\bar{z}}^{-1} .\right.\right.
\end{aligned}
$$

Remark 4. The usage of a singularly perturbed operator together with its adjoint one allows to provide additional description of both operators as one whole object. Namely, linear unbounded closed operator $\tilde{A} \neq A$, densely defined in $\mathcal{H}$, is singularly perturbed $\mathcal{H}_{-1}$-class with respect to the operator $A$ (without loss of generality $0 \in \rho(A) \cap \rho(\tilde{A})$ ), if sets

$$
\mathfrak{D}=\{f \in \mathfrak{D}(A) \cap \mathfrak{D}(\tilde{A}): A f=\tilde{A} f\}, \quad \mathfrak{D}_{*}=\left\{f \in \mathfrak{D}\left(A^{*}\right) \cap \mathfrak{D}\left((\tilde{A})^{*}\right): A^{*} f=\tilde{A}^{*} f\right\}
$$

are dense in $\mathcal{H}$ both, herewith $\mathfrak{D} \subset \mathcal{H}_{+1}, \mathfrak{D}_{*} \subset \mathcal{H}_{+1}$.

It is clear that for each couple $A, \tilde{A}$ and $A^{*}, \tilde{A}^{*}$ there exist joint restrictions i.e. operators $\dot{A}:=A\left\lceil\mathfrak{D}\right.$ and $\dot{A}_{*}:=A\left\lceil\mathfrak{D}_{*}\right.$, so that each of them has nontrivial deficiency indices

$$
\mathbf{n}^{ \pm}(\dot{A})=\operatorname{dim} \operatorname{ker}(\dot{A} \mp z)^{*} \neq 0, \quad \mathbf{n}^{ \pm}\left(\dot{A}_{*}\right)=\operatorname{dim} \operatorname{ker}\left(\dot{A}_{*} \mp z\right)^{*} \neq 0, \quad z \in \rho(A) .
$$

In this paper we consider only the case $\mathbf{n}^{ \pm}(\dot{A})=\mathbf{n}^{ \pm}\left(\dot{A}_{*}\right)=1$.

The last description is close to the topic of solvable extensions described in the work of M.I. Vishik [12]. If $\mathfrak{D}=\mathfrak{D}_{*}$ and $\tilde{A}=\tilde{A}^{*}$, then we have usual description of singularly perturbed self-adjoint operator $[3,10]$.

The perturbed operator can possess a new point $\lambda$ of the point spectrum $\sigma_{p}(\tilde{A})$, which does not exist in $\sigma_{p}(A)$.

Proposition 1. If $\tilde{A} \in \mathcal{P}_{-1}^{1}(A)$ possesses new eigenvalue $\lambda \in \mathbb{C}$ in comparison with $A$, namely there exists $\lambda \in \sigma_{p}(\tilde{A})$, but $\lambda \notin \sigma_{p}(A)$, then for corresponding eigenvectors $\varphi$ : $\tilde{A} \varphi=\lambda \varphi$ and $\psi: \tilde{A}^{*} \psi=\bar{\lambda} \psi$, we have equalities:

$$
\begin{aligned}
(\lambda-z) b_{z}\left(\varphi,\left(\mathbf{A}^{*}-\bar{z}\right)^{-1} \omega_{1}\right)=1, & \varphi=(\mathbf{A}-\lambda)^{-1} \omega_{2}, \\
(\bar{\lambda}-\bar{z}) \bar{b}_{z}\left(\psi,(\mathbf{A}-z)^{-1} \omega_{2}\right) & =1, \quad \psi=\left(\mathbf{A}^{*}-\bar{\lambda}\right)^{-1} \omega_{1},
\end{aligned}
$$

where $\eta_{i}(z)(i \in\{1,2\})$ is defined in Theorem $1, z \in \rho(A) \bigcap \rho(\tilde{A})$.

Proof. Solving the eigenvalue problem in the form $\tilde{R}_{z} \varphi=\frac{1}{\lambda-z} \varphi$ and using expressions for the resolvents (6) and also (10), we obtain (16). Indeed,

$$
\begin{gathered}
\tilde{R}_{z} \varphi=R_{z} \varphi+b_{z}\left(\varphi, \eta_{1}(\bar{z})\right) \eta_{2}(z)=\frac{1}{\lambda-z} \varphi \\
b_{z}\left(\varphi, \eta_{1}(\bar{z})\right) \eta_{2}(z)=\frac{1}{\lambda-z} \varphi-R_{z} \varphi=\frac{1}{\lambda-z}(A-\lambda)(A-z)^{-1} \varphi .
\end{gathered}
$$


Hence, $(\lambda-z) b_{z}\left(\varphi, \eta_{1}(\bar{z})\right)(A-z)(A-\lambda)^{-1} \eta_{2}(z)=\varphi$. The multiplication on $\eta_{1}(\bar{z})$ gives $(\lambda-z) b_{z}\left(\varphi, \eta_{1}(\bar{z})\right)=1$, if we put $(A-z)(A-\lambda)^{-1} \eta_{2}(z)=\varphi=(\mathbf{A}-\lambda)^{-1} \omega_{2}$. Then we obtain $(16)$.

Expressions (16) are obtained analogously. In the case of adjoint operator (17), we write the expression adjoint to (6) and (7). Indeed,

$$
\begin{gathered}
\tilde{R}_{z}^{*} \varphi=R_{z}^{*} \psi+b_{z}\left(\psi, \eta_{2}(z)\right) \eta_{1}(\bar{z})=\frac{1}{\bar{\lambda}-\bar{z}} \psi, \\
\bar{b}_{z}\left(\psi, \eta_{2}(z)\right) \eta_{1}(\bar{z})=\frac{1}{\bar{\lambda}-\bar{z}} \varphi-R_{z}^{*} \psi=\frac{1}{\bar{\lambda}-\bar{z}}\left(A^{*}-\bar{\lambda}\right)\left(A^{*}-\bar{z}\right)^{-1} \psi .
\end{gathered}
$$

Hence, $(\bar{\lambda}-\bar{z}) b_{\bar{z}}\left(\psi, \eta_{2}(z)\right)\left(A^{*}-\bar{z}\right)\left(A^{*}-\bar{\lambda}\right)^{-1} \eta_{1}(\bar{z})=\psi$. The multiplication on $\eta_{2}(z)$ gives $(\bar{\lambda}-\bar{z}) b_{\bar{z}}\left(\psi, \eta_{2}(z)\right)=1$, if we put $\left(A^{*}-\bar{z}\right)\left(A^{*}-\bar{\lambda}\right)^{-1} \eta_{1}(\bar{z})=\psi=\left(\mathbf{A}^{*}-\bar{\lambda}\right)^{-1} \omega_{1}$. Then we obtain (17).

The inverse spectral problem is also solvable for $\tilde{A} \in \mathcal{P}_{-1}^{1}(A)$.

Theorem 2. For a given linear unbounded closed operator $A$ in the separable Hilbert space $\mathcal{H}$, numbers $\lambda \in \mathbb{C}$ and vectors $\varphi, \psi \in \mathcal{H}_{+1} \backslash \mathcal{H}_{+2}$, such that for some $z \in \rho(A), z \neq \lambda$ and $\left(\varphi,\left(A^{*}-\bar{\lambda}\right)\left(A^{*}-\bar{z}\right)^{-1} \psi\right) \neq 0$, there exists unique singularly non-symmetrically perturbed rank one operator $\tilde{A} \in \mathcal{P}_{-1}^{1}(A)$ such that $\lambda, \bar{\lambda}$ and $\varphi, \psi$ are its eigenvalues and eigenvectors respectively: namely, $\tilde{A} \varphi=\lambda \varphi$ and $\tilde{A}^{*} \psi=\bar{\lambda} \psi$. Herewith, the operator $\tilde{A}$ is defined due to the resolvent (6) from Theorem 1:

$$
\tilde{R}_{z}=R_{z}+b_{z}\left(\cdot, \eta_{1}(\bar{z})\right) \eta_{2}(z)
$$

with the vector-valued functions

$$
\eta_{1}(\bar{z})=\left(A^{*}-\bar{\lambda}\right)\left(A^{*}-\bar{z}\right)^{-1} \psi, \eta_{2}(z)=(A-\lambda)(A-z)^{-1} \varphi,
$$

and with the scalar-valued function

$$
b_{z}^{-1}=(\lambda-z)\left(\varphi, \eta_{1}(\bar{z})\right), \quad\left(\bar{b}_{z}^{-1}=(\bar{\lambda}-\bar{z})\left(\psi, \eta_{2}(z)\right)\right) .
$$

Remark 5. The inequality $\left(\varphi,\left(A^{*}-\bar{\lambda}\right)\left(A^{*}-\bar{z}\right)^{-1} \psi\right) \neq 0$ is equivalent to the inequality $\left(\psi,(A-\lambda)(A-z)^{-1} \varphi\right) \neq 0$

Proof of Remark 5. Indeed,

$$
\begin{gathered}
\left(\psi,(A-\lambda)(A-z)^{-1} \varphi\right) \neq 0 \Longleftrightarrow \overline{\left(\psi,(A-\lambda)(A-z)^{-1} \varphi\right)} \neq 0 \Longleftrightarrow \\
\left((A-\lambda)(A-z)^{-1} \varphi, \psi\right) \neq 0 \Longleftrightarrow\left(\varphi,\left(A^{*}-\bar{\lambda}\right)\left(A^{*}-\bar{z}\right)^{-1} \psi\right) \neq 0 .
\end{gathered}
$$

Proof of Theorem 2. Let us start to prove Theorem 2. At once, it is possible to show that both sets

$$
\mathfrak{D}=\left\{f \in \mathfrak{D}(A):\left(\left(A^{*}-\bar{z}\right) f, \eta_{2}(z)\right)=0\right\}, \quad \mathfrak{D}_{*}=\left\{f \in \mathfrak{D}(A):\left((A-z) f, \eta_{1}(\bar{z})\right)=0\right\}
$$

coincide with written one in (15) and are dense in $\mathcal{H}$, respectively. If we suppose $\exists h \in \mathcal{H}$ such that $(\mathfrak{D}, h)=0$, then

$$
0=(\mathfrak{D}, h)=\left((A-z)^{-1} \mathfrak{M}_{z}, h\right)=\left(\mathfrak{M}_{z},\left(A^{*}-\bar{z}\right)^{-1} h\right)
$$


namely $\left(A^{*}-\bar{z}\right)^{-1} h \in \mathfrak{D}\left(A^{*}\right)$ and $\left(A^{*}-\bar{z}\right)^{-1} h \in \mathfrak{N}_{z} \perp \mathfrak{M}_{z}$, but $\mathfrak{N}_{z} \cap \mathfrak{D}\left(A^{*}\right)=\{0\}$.

Analogously, if $\exists h \in \mathcal{H}$ such that $\left(\mathfrak{D}_{*}, h\right)=0$, then

$$
0=\left(\mathfrak{D}_{*}, h\right)=\left(\left(A^{*}-\bar{z}\right)^{-1} \mathfrak{M}_{\bar{z}}, h\right)=\left(\mathfrak{M}_{z},(A-z)^{-1} h\right),
$$

namely $(A-z)^{-1} h \in \mathfrak{D}_{*}(A)$ and $(A-z)^{-1} h \in \mathfrak{N}_{\bar{z}} \perp \mathfrak{M}_{\bar{z}}$, but $\mathfrak{N}_{\bar{z}} \cap \mathfrak{D}_{*}(A)=\{0\}$.

Further proof of Theorem 2 is essentially based on the next technical proposition.

Proposition 2. Let $A$ be a linear unbounded closed operator in a separable Hilbert space $\mathcal{H}$. Then, the operator-valued function

$$
\tilde{R}_{z}:=(A-z)^{-1}+b_{z}\left(\cdot, \eta_{1}(\bar{z})\right) \eta_{2}(z), \quad z \in \rho(A),
$$

is a resolvent of some linear closed operator, if for $\eta_{1}(z), \eta_{2}(z)$ and $b(z)$ we have expressions

$$
\begin{gathered}
\eta_{1}(\bar{z})=\left(A^{*}-\bar{\xi}\right)\left(A^{*}-\bar{z}\right)^{-1} \eta_{1}(\bar{\xi}), \quad \eta_{2}(z)=(A-\xi)(A-z)^{-1} \eta_{2}(\xi), \\
b_{z}^{-1}-b_{\xi}^{-1}=(\xi-z)\left(\eta_{2}(\xi), \eta_{1}(\bar{z})\right), \quad z, \xi \in \rho(A) \cap \rho(\tilde{A}) ;
\end{gathered}
$$

and additionally it is a resolvent of a singularly non-symmetrically rank one perturbed operator of $\mathcal{H}_{-1}$-class, if $\eta_{1}(z), \eta_{2}(z) \in \mathcal{H}_{+1} \backslash \mathcal{H}_{+2}$.

Proof. The proof of Proposition 2 is based on Theorems 1 and 2 from [9, Ch. VIII, §1]. Namely, the operator-valued function $(21) \tilde{R}_{z}$ is a resolvent of some closed operator if:

a) $\tilde{R}_{z}$ satisfies the Hilbert identity with some $z, \xi \in \mathbb{C}: \tilde{R}_{z}-\tilde{R}_{\xi}=(z-\xi) \tilde{R}_{z} \tilde{R}_{\xi}$, namely it is so called pseudoresolvent, and

b) $\tilde{R}_{z}$ has the trivial kernel, i.e. $\operatorname{ker}\left(\tilde{R}_{z}\right)=\{0\}$.

We will verify the condition a). Substitute the operator-valued function, defined in (6), into the Hilbert identity:

$$
\begin{aligned}
& \left.\left((A-z)^{-1}+b_{z}\left(\cdot, \eta_{1}(\bar{z})\right)\right) \eta_{2}(z)\right)-\left((A-\xi)^{-1}+b_{\xi}\left(\cdot, \eta_{1}(\bar{\xi})\right) \eta_{2}(\xi)\right)= \\
= & \left.(z-\xi)\left((A-z)^{-1}+b_{z}\left(\cdot, \eta_{1}(\bar{z})\right)\right) \eta_{2}(z)\right)\left((A-\xi)^{-1}+b_{\xi}\left(\cdot, \eta_{1}(\bar{\xi})\right) \eta_{2}(\xi)\right) .
\end{aligned}
$$

Using more the Hilbert identity for $(A-z)^{-1}$, we obtain

$$
\begin{array}{r}
\left.b_{z}\left(\cdot, \eta_{1}(\bar{z})\right)\right) \eta_{2}(z)-b_{\xi}\left(\cdot, \eta_{1}(\bar{\xi})\right) \eta_{2}(\xi)=(z-\xi) b_{\xi}\left(\cdot, \eta_{1}(\bar{\xi})\right)(A-z)^{-1} \eta_{2}(\xi)+ \\
\left.+(z-\xi) b_{z}\left(\cdot,\left(A^{*}-\bar{\xi}\right)^{-1} \eta_{1}(\bar{z})\right) \eta_{2}(z)+(z-\xi) b_{z} b_{\xi}\left(\cdot, \eta_{1}(\bar{\xi})\right)\left(\eta_{2}(\xi), \eta_{1}(\bar{z})\right)\right) \eta_{2}(z) .
\end{array}
$$

From the second equality (22) we get

$$
\eta_{2}(z)=\eta_{2}(\xi)+(z-\xi)(A-z)^{-1} \eta_{2}(\xi), \quad(z-\xi)(A-z)^{-1} \eta_{2}(\xi)=\eta_{2}(z)-\eta_{2}(\xi) .
$$

By analogy, from the second equality (22), we obtain

$$
(\bar{z}-\bar{\xi})\left(A^{*}-\bar{\xi}\right)^{-1} \eta_{1}(\bar{z})=\eta_{1}(\bar{z})-\eta_{1}(\bar{\xi}) .
$$

Substitute two last equalities in (24):

$$
\begin{gathered}
b_{z}\left(\cdot, \eta_{1}(\bar{z})\right) \eta_{2}(z)-b_{\xi}\left(\cdot, \eta_{1}(\bar{\xi})\right) \eta_{2}(\xi)=b_{\xi}\left(\cdot, \eta_{1}(\bar{\xi})\right)\left[\eta_{2}(z)-\eta_{2}(\xi)\right]+ \\
\left.+b_{z}\left(\cdot,\left[\eta_{1}(\bar{z})-\eta_{1}(\bar{\xi})\right]\right) \eta_{2}(z)+(z-\xi) b_{z} b_{\xi}\left(\cdot, \eta_{1}(\bar{\xi})\right)\left(\eta_{2}(\xi), \eta_{1}(\bar{z})\right)\right) \eta_{2}(z) .
\end{gathered}
$$


Simplifying last expression we obtain

$$
\left.0=b_{\xi}\left(\cdot, \eta_{1}(\bar{\xi})\right) \eta_{2}(z)-b_{z}\left(\cdot, \eta_{1}(\bar{\xi})\right) \eta_{2}(z)+(z-\xi) b_{z} b_{\xi}\left(\cdot, \eta_{1}(\bar{\xi})\right)\left(\eta_{2}(\xi), \eta_{1}(\bar{z})\right)\right) \eta_{2}(z) .
$$

After reduction on $\left(\cdot, \eta_{1}(\bar{\xi})\right) \eta_{2}(z)$ we obtain the expression, that gives $(23)$.

Verify the condition b). For a vector $f \in \mathcal{H}$, such that $f \perp \eta_{1}(\bar{z})$, with a fixed $z$, the vector-valued function $\tilde{R}(z)=(A-z)^{-1}$ is a resolvent of some closed operator, since for such vector we have $\tilde{R}(z) f=R(z) f=(A-z)^{-1} f$.

For the vector $\eta_{1}(\bar{z})$ we have $\tilde{R}_{z} \eta_{1}(\bar{z})=(A-z)^{-1} \eta_{1}(\bar{z})+b_{z}\left(\eta_{1}(\bar{z}), \eta_{1}(\bar{z})\right) \eta_{2}(z) \neq 0$, since, if $(A-z)^{-1} \eta_{1}(\bar{z})=-b_{z}\left(\eta_{1}(\bar{z}), \eta_{1}(\bar{z})\right) \eta_{2}(z)$, then from $\eta_{1}(\bar{z}) \in \mathcal{H}_{+1} \backslash \mathcal{H}_{+2}$ we would have $\eta_{2}(z) \in \mathcal{H}_{+2}$, but by the conditions of Proposition we have $\eta_{2}(z) \notin \mathcal{H}_{+2}$.

By analogy, for a vector $f \in \mathcal{H}$, such that $f \perp \eta_{2}(z)$ with a fixed $z$, the expression $\left(\tilde{R}_{z}\right)^{*}=\left(\tilde{A}^{*}-\bar{z}\right)^{-1}$ is a resolvent of some closed operator, since for such a vector we have $\left(\tilde{R}_{z}\right)^{*} f=R_{z}^{*} f=(A-\bar{z})^{-1} f$.

For the vector $\eta_{2}(z)$ we have $\left(\tilde{R}_{z}\right) \eta_{2}(z)=(A-\bar{z})^{-1} \eta_{2}(z)+\bar{b}_{z}\left(\eta_{2}(z), \eta_{2}(z)\right) \eta_{1}(\bar{z}) \neq 0$, since, if it would be $(A-\bar{z})^{-1} \eta_{2}(z)=-\bar{b}_{z}\left(\eta_{2}(z), \eta_{2}(z)\right) \eta_{1}(\bar{z})$, then from $\eta_{2}(z) \in \mathcal{H}_{+1}$, we would have $\eta_{1}(\bar{z}) \in \mathcal{H}_{+2}$, but by the assumptions of Proposition 2 we have $\eta_{1}(\bar{z}) \notin \mathcal{H}_{+2}$.

And now, we can say that there exists a closed linear operator $\tilde{A}$, and we can put $(\tilde{A}-z)^{-1}:=\tilde{R}(z), z \in \rho(\tilde{A})$.

Taking into account Theorem 1 , we obtain that the defined in (6) $\tilde{R}(z)$ is the resolvent of singularly non-symmetrically perturbed of rank one operators $\mathcal{H}_{-1}$-class.

Now we can continue the proof of Theorem 2 by steps.

1. If $\varphi, \psi \in \mathcal{H}_{+1} \backslash \mathcal{H}_{+2}$, then from (19) it follows that $\eta_{1}(z), \eta_{2}(z) \in \mathcal{H}_{+1} \backslash \mathcal{H}_{+2}$. Proposition 2 gives that the defined in (21) operator $\tilde{R}_{z}$ is a resolvent of some closed operator under the condition $\eta_{1}(z), \eta_{2}(z)$ satisfy $(22)$ and $b_{z}$ satisfies $(23)$.

Let for $\eta_{1}(z), \eta_{2}(z)$ we have (19), i.e.

$$
\begin{aligned}
& \eta_{2}(z)=(A-\lambda)(A-z)^{-1} \varphi=\varphi+(z-\lambda)(A-z)^{-1} \varphi \\
& \eta_{1}(\bar{z})=(A-\bar{\lambda})(A-\bar{z})^{-1} \psi=\psi+(\bar{z}-\bar{\lambda})(A-\bar{z})^{-1} \psi
\end{aligned}
$$

and for $b_{z}$ we have $(20): b_{z}^{-1}=(\lambda-z)\left(\varphi, \eta_{1}(\bar{z})\right), \quad \bar{b}_{z}^{-1}=(\bar{\lambda}-\bar{z})\left(\psi, \eta_{2}(z)\right)$, which we write in the form

$$
b_{z}^{-1}=(\lambda-z) \overline{\left(\psi, \eta_{2}(z)\right)}, \quad b_{z}^{-1}=(\lambda-z)\left(\eta_{2}(z), \psi\right) .
$$

Write the left-hand side of (23) using (25) and (26):

$$
\begin{gathered}
b_{z}-b_{\xi}=(\lambda-z)\left(\varphi,\left[\psi+(\bar{z}-\bar{\lambda})\left(A^{*}-\bar{z}\right)^{-1} \psi\right]\right)-(\lambda-\xi)\left(\left[\varphi+(\xi-\lambda)(A-\xi)^{-1} \varphi\right], \psi\right)= \\
=(\lambda-z)(\varphi, \psi)-(\lambda-z)^{2}\left((A-z)^{-1} \varphi, \psi\right)-(\lambda-\xi)(\varphi, \psi)+(\lambda-\xi)^{2}\left((A-\xi)^{-1} \varphi, \psi\right)= \\
=(\xi-z)(\varphi, \psi)-(\lambda-z)^{2}\left((A-z)^{-1} \varphi, \psi\right)+(\lambda-\xi)^{2}\left((A-\xi)^{-1} \varphi, \psi\right) .
\end{gathered}
$$

Write the right-hand side of (23) using (25):

$$
\begin{aligned}
&(\xi-z)\left(\eta_{2}(z), \eta_{1}(\bar{z})\right)=(\xi-z)\left(\left[\varphi+(\xi-\lambda)(A-\xi)^{-1} \varphi\right],\left[\psi+(\bar{z}-\bar{\lambda})\left(A^{*}-\bar{z}\right)^{-1} \psi\right]\right)= \\
&=(\xi-z)\left[(\varphi, \psi)+(z-\lambda)\left((A-z)^{-1} \varphi, \psi\right)+\right. \\
&\left.+(\xi-\lambda)\left((A-\xi)^{-1} \varphi, \psi\right)+(z-\lambda)(\xi-\lambda)\left((A-z)^{-1}(A-\xi)^{-1} \varphi, \psi\right)\right] .
\end{aligned}
$$


In the last line we used the Hilbert identity: $(A-z)^{-1}-(A-\xi)^{-1}=(z-\xi)(A-z)^{-1}(A-\xi)^{-1}$. Hence,

$$
\begin{gathered}
(\xi-z)\left(\eta_{2}(\xi), \eta_{1}(\bar{z})\right)=(\xi-z)(\varphi, \psi)+(\xi-z)(z-\lambda)\left((A-z)^{-1} \varphi, \psi\right)+ \\
+(\xi-z)(\xi-\lambda)\left((A-\xi)^{-1} \varphi, \psi\right)+(\xi-\lambda)(z-\lambda)\left((A-\xi)^{-1} \varphi, \psi\right)- \\
-(z-\lambda)(\xi-\lambda)\left((A-z)^{-1} \varphi, \psi\right)=(\xi-z)(\varphi, \psi)+\left(-z^{2}+z \xi-\lambda \xi+\lambda z\right)\left((A-z)^{-1} \varphi, \psi\right)+ \\
+\left(\xi^{2}-z \xi-\lambda \xi+\lambda z\right)\left((A-\xi)^{-1} \varphi, \psi\right)+\left(z \xi-\lambda z-\lambda \xi+\lambda^{2}\right)\left((A-\xi)^{-1} \varphi, \psi\right)- \\
-\left(z \xi-\lambda z-\lambda \xi+\lambda^{2}\right)\left((A-z)^{-1} \varphi, \psi\right)= \\
=(\xi-z)(\varphi, \psi)-\left(\lambda^{2}-2 \lambda z+z^{2}\right)\left((A-z)^{-1} \varphi, \varphi\right)+\left(\xi^{2}-2 \lambda \xi+\lambda^{2}\right)\left((A-\xi)^{-1} \varphi, \psi\right) .
\end{gathered}
$$

Therefore,

$$
(\xi-z)\left(\eta_{2}(\xi), \eta_{1}(\bar{z})\right)=(\xi-z)(\varphi, \psi)+(\xi-\lambda)^{2}\left((A-\xi)^{-1} \varphi, \psi\right)-(z-\lambda)^{2}\left((A-z)^{-1} \varphi, \psi\right) .
$$

Comparing (27) and (28) we obtain (23).

2. Since vectors $\eta_{1}(z)$ and $\eta_{2}(z)$ belong to $\mathcal{H}_{+1} \backslash \mathcal{H}_{+2}$, by Proposition 2 the operator $\tilde{A}$ is a singular perturbation of $A$.

3. Let us prove $\tilde{A} \varphi=\lambda \varphi$ in the form $(\tilde{A}-z)^{-1} \varphi=\frac{1}{\lambda-z} \varphi$.

Indeed, the substitution of the first expression (20) and first expression of (25) in (18) gives

$$
(\tilde{A}-z)^{-1} \varphi=(A-z)^{-1} \varphi+\frac{1}{(\lambda-z)\left(\varphi, \eta_{1}(\bar{z})\right)}\left(\varphi, \eta_{1}(\bar{z})\right)\left(\varphi+(z-\lambda)(A-z)^{-1} \varphi\right)=\frac{1}{\lambda-z} \varphi .
$$

Show $\tilde{A}^{*} \psi=\bar{\lambda} \psi$ in the form $\left(\tilde{A}^{*}-z\right)^{-1} \psi=\frac{1}{\lambda-\bar{z}} \psi$. Analogously, the substitution the second expression of (20) and the second expression of (25) in a joint to (18) give us $\left(\tilde{A}^{*}-\bar{z}\right)^{-1} \psi=(A-\bar{z})^{-1} \psi+\frac{1}{(\bar{\lambda}-\bar{z})\left(\psi, \eta_{2}(z)\right)}\left(\psi, \eta_{2}(z)\right)\left(\psi+(\bar{z}-\bar{\lambda})\left(A^{*}-\bar{z}\right)^{-1} \psi\right)=\frac{1}{\bar{\lambda}-\bar{z}} \psi$.

4. Show the uniqueness. Suppose the contrary i.e. there exists another operator $\hat{A} \neq \tilde{A}$ such that $\hat{A} \varphi=\lambda \varphi$ and $\hat{A}^{*} \psi=\bar{\lambda} \psi$. Since $\hat{A} \in \mathcal{P}(A)$ is also singularly non-symmetrically perturbed of rank one operator $A$, due to Theorem 1 it has the representation

$$
(\hat{A}-z)^{-1}:=(A-z)^{-1}+\hat{b}_{z}\left(\cdot, \hat{\eta}_{1}(\bar{z})\right) \hat{\eta}_{2}(z), z \in \rho(\hat{A}) \cap \rho(A)
$$

where at least one of inequalities $\hat{\eta}_{1}(z) \neq \eta_{1}(z)$ either $\hat{\eta}_{2}(z) \neq \eta_{2}(z)$ or $\hat{b}_{z} \neq b_{z}$ is true. Then for $\lambda$ and $\varphi$ we have

$$
\begin{gathered}
(\hat{A}-z)^{-1} \varphi=(\tilde{A}-z)^{-1} \varphi=\frac{1}{\lambda-z} \varphi= \\
=(A-z)^{-1} \varphi+\hat{b}_{z}\left(\varphi, \hat{\eta}_{1}(\bar{z})\right) \hat{\eta}_{2}(z)=(A-z)^{-1} \varphi+b_{z}\left(\varphi, \eta_{1}(\bar{z})\right) \eta_{2}(z),
\end{gathered}
$$

namely

$$
\hat{b}_{z}\left(\varphi, \hat{\eta}_{1}(\bar{z})\right) \hat{\eta}_{2}(z)=b_{z}\left(\varphi, \eta_{1}(\bar{z})\right) \eta_{2}(z) .
$$

From the last equality we deduce $\eta_{2}(z)=\mu \hat{\eta}_{2}(z)$ for a fixed $z$, i.e. $b_{z}\left(\varphi, \eta_{1}(\bar{z})\right)=\mu \hat{b}_{z}\left(\varphi, \hat{\eta}_{1}(\bar{z})\right)$.

Analogously, for $\bar{\lambda}$ and $\psi$ we have

$$
\begin{gathered}
\left(\hat{A}^{*}-\bar{z}\right)^{-1} \psi=\left(\tilde{A}^{*}-\bar{z}\right)^{-1} \psi=\frac{1}{\bar{\lambda}-\bar{z}} \psi= \\
=\left(A^{*}-\bar{z}\right)^{-1} \psi+\overline{\hat{b}}_{z}\left(\psi, \hat{\eta}_{2}(z)\right) \hat{\eta}_{1}(\bar{z})=\left(A^{*}-\bar{z}\right)^{-1} \psi+\bar{b}_{z}\left(\psi, \eta_{2}(z)\right) \eta_{1}(\bar{z}),
\end{gathered}
$$


namely $\overline{\hat{b}}_{z}\left(\psi, \hat{\eta}_{2}(z)\right) \hat{\eta}_{1}(\bar{z})=\bar{b}_{z}\left(\psi, \eta_{2}(z)\right) \eta_{1}(\bar{z})$.

The last expression gives $\hat{\eta}_{1}(\bar{z})=\nu \eta_{1}(\bar{z})$ for fixed $z$. Now (30) yields

$$
b_{z}\left(\varphi, \eta_{1}(\bar{z})\right) \eta_{2}(z)=\hat{b}_{z}\left(\varphi, \nu \eta_{1}(\bar{z})\right) \mu \eta_{2}(z)=\hat{b}_{z} \bar{\nu} \mu\left(\varphi, \eta_{1}(\bar{z})\right) \eta_{2}(z)
$$

namely $b_{z}=\hat{b}_{z} \bar{\nu} \mu$ and hence $\hat{b}_{z}=b_{z} \frac{1}{\bar{\nu} \mu}$. And (29) has a form

$$
(\hat{A}-z)^{-1}:=(A-z)^{-1}+\frac{1}{\bar{\nu} \mu}\left(\cdot, \nu \eta_{1}(\bar{z})\right) \mu \eta_{2}(z)=(\tilde{A}-z)^{-1} .
$$

The obtained contradiction finishes the proof of the uniqueness and the proof of the theorem in general.

Remark 6. Theorem 2, in fact, states more than the statement inverse to Proposition 1 . In Proposition 1 we have $\lambda \in \sigma_{p}(\tilde{A}), \lambda \in \rho(A)$, simultaneously, in Theorem $2 \lambda \in \sigma_{p}(\tilde{A})$ can be immersed in the continuous spectrum $\sigma_{c}(A)$, and hence in $\sigma_{c}(\tilde{A})$.

3. Perturbations $\mathcal{H}_{-2}$-class. Transfer the results of the previous paragraph to the perturbation of the $\mathcal{H}_{-2}$-class. This general case requires an additional parametrization. Then Definition 1 takes a slightly different form.

Definition 2. Let $A$ be a linear unbounded closed operator in a separable Hilbert space $\mathcal{H}$. For $\omega_{1}, \omega_{2} \in \mathcal{H}_{-2}$, where at least one of the vectors $\omega_{1}, \omega_{2}$ does not belong to $\mathcal{H}_{-1}$, we put $\eta_{1}(\bar{z})=\left(\mathbf{A}^{*}-\bar{z}\right)^{-1} \omega_{1}, \eta_{2}(z)=(\mathbf{A}-z)^{-1} \omega_{2}, z \in \rho(\mathbf{A})$. The operator $\tilde{A} \in \mathcal{P}_{-2}^{1}(A)$ is called singularly non-symmetrically rank one perturbed of the $\mathcal{H}_{-2}$-class with respect to the operator $A$, if its domain is defined (by fixed $z$ )

$$
\begin{aligned}
& \mathfrak{D}(\tilde{A})=\left\{\vartheta=\phi-\mathbf{b}_{z} \eta_{2}(z): \phi \in \mathfrak{D}(A),\right. \\
& \left.\mathbf{b}_{z}=\frac{\left((A-z) \phi, \eta_{1}(\bar{z})\right)}{1 / \alpha+\tau+\left((A-z)(1+z A)\left(A^{2}+1\right)^{-1} \eta_{2}(z), \eta_{1}(\bar{z})\right)}\right\}
\end{aligned}
$$

in the case $\left((A-z)(1+z A)\left(A^{2}+1\right)^{-1} \eta_{2}(z), \eta_{1}(\bar{z})\right)+1 / \alpha+\tau \neq 0$, where $\tau \in \mathbb{C}$ is a parameter; and

$$
\mathfrak{D}(\tilde{A})=\mathfrak{D}_{\mathcal{H}_{2}} \dot{+}\left\{c \eta_{2}(z)\right\}, \quad \mathfrak{D}_{\mathcal{H}_{2}}=\left\{\phi \in \mathfrak{D}(A): \quad\left((A-z) \phi, \eta_{1}(\bar{z})\right)=0\right\}, c \in \mathbb{C},
$$

in the case $\left((A-z)(1+z A)\left(A^{2}+1\right)^{-1} \eta_{2}(z), \eta_{1}(\bar{z})\right)+1 / \alpha+\tau=0$; the action is defined by the rule

$$
(\tilde{A}-z) \vartheta=(A-z) \phi .
$$

Note at once that the remark similar to 1 is not possible at all in the case of Definition 2 due to the stronger singularity of the perturbation.

Remark 7. Similarly to Remark 2 in Definition 2 two cases for the domain $\mathfrak{D}(\tilde{A})$ in $(31)$ and (32) are due to the fact that $z \notin \sigma_{p}(\tilde{A})$ in the case $(31)$ and $z \in \sigma_{p}(\tilde{A})$ in the case $(32)$.

Remark 8. If $A$ is a self-adjoint operator, $\omega_{1}=\omega_{2}$, and $\alpha \in \mathbb{R} \backslash\{0\}$ in Definition 2, then we obtain the usual known definition of singularly perturbed self-adjoint $\mathcal{H}_{-2}$-class operator $[3,10]$.

For the resolvent $R_{z}, \quad z \in \rho(A)$ of an unperturbed operator $A$ and the resolvent $\tilde{R}_{z}$, $z \in \rho(\tilde{A})$ of perturbed $\mathcal{H}_{-2}$-class operator $\tilde{A}$ we have also a theorem similar to Theorem 1 . 
Theorem 3. Let $A$ be a linear unbounded closed operator in the separable Hilbert space $\mathcal{H}$ and $\tilde{A}$ be its singularly non-symmetrically rank one perturbed with respect to $A$ operator $\tilde{A} \in \mathcal{P}_{-2}^{1}(A)$ defined by (31)-(33). Then the resolvents $R_{z}$ and $\tilde{R}_{z}$ are related to each other by the M. Krein type formula:

$$
\tilde{R}_{z}=R_{z}+b_{z}\left(\cdot, \eta_{1}(\bar{z})\right) \eta_{2}(z),
$$

with vector-valued functions $\eta_{1}(z)=(A-\xi)(A-z)^{-1} \eta_{1}(\xi), \eta_{2}(z)=(A-\xi)(A-z)^{-1} \eta_{2}(\xi)$, where $\eta_{1}(z), \eta_{2}(z) \in \mathcal{H}, z, \xi \in \rho(A) \cap \rho(\tilde{A})$, and with the scalar-valued function

$$
-b_{z}^{-1}=\alpha^{-1}+\tau+\left((A-z)(1+z A)\left(A^{2}+1\right)^{-1} \eta_{2}(z), \eta_{1}(\bar{z})\right),
$$

where $\alpha \in \mathbb{C}, 0<|\alpha|<\infty$ and $\forall \tau \in \mathbb{C}$, for which we have the equality:

$$
b_{z}^{-1}-b_{\xi}^{-1}=(\xi-z)\left(\eta_{2}(\xi), \eta_{1}(\bar{z})\right),
$$

and vectors $\eta_{1}(z), \eta_{2}(z)$ are connected to $\omega_{1}, \omega_{2}$ by relations $\eta_{1}(\bar{z})=\left(\mathbf{A}^{*}-\bar{z}\right)^{-1} \omega_{1}, \eta_{2}(z)=$ $(\mathbf{A}-z)^{-1} \omega_{2}$.

Analogously to Theorem 1 , in general, the case $\alpha=0$ can also be considered by assigning $b_{z} \equiv 0 \tilde{R}_{z} \equiv R_{z}$. One can put also $|\alpha|=\infty$, then in the last expression (35) the term $\alpha^{-1}$ will be omitted.

Proof of Theorem 3. Steps of the proof are generally similar to those of Theorem 1, but differ in the technical details that arise due to perturbations of $\mathcal{H}_{-2}$-class.

For $\forall h \in \mathcal{H}, z \in \rho(\tilde{A}) \cap \rho(A),(\tilde{A}-z)^{-1} h=\psi \in \mathfrak{D}(\tilde{A})$. Hence, we can put $(A-z)^{-1} h=\varphi$ and substitute in $(34)$ :

$$
\begin{gathered}
\psi=(\tilde{A}-z)^{-1} h=(A-z)^{-1} h+b_{z}\left(h, \eta_{1}(\bar{z})\right) \eta_{2}(z)= \\
=\varphi-\frac{1}{1 / \alpha+\tau+\left((A-z)(1+z A)\left(A^{2}+1\right)^{-1} \eta_{2}(z), \eta_{1}(\bar{z})\right)}\left(h, \eta_{1}(\bar{z})\right) \eta_{2}(z)= \\
=\varphi-\frac{\left((A-z) \varphi, \eta_{1}(\bar{z})\right)}{1 / \alpha+\tau+\left((A-z)(1+z A)\left(A^{2}+1\right)^{-1} \eta_{2}(z), \eta_{1}(\bar{z})\right)} \eta_{2}(z) .
\end{gathered}
$$

Comparing the start and the end of the last expression, we obtain (31).

Let us show (36). Using the equality (35), we have

$$
\begin{gathered}
b_{z}^{-1}-b_{\xi}^{-1}=-\left((A-z)(1+z A)\left(A^{2}+1\right)^{-1} \eta_{2}(z), \eta_{1}(\bar{z})\right)+ \\
+\left((A-\xi)(1+\xi A)\left(A^{2}+1\right)^{-1} \eta_{2}(\xi), \eta_{1}(\bar{\xi})\right) .
\end{gathered}
$$

And we use the following equalities

$$
\eta_{1}(\bar{\xi})=(A-\bar{z})(A-\bar{\xi})^{-1} \eta_{1}(\bar{z}), \eta_{2}(z)=(A-\xi)(A-z)^{-1} \eta_{2}(\xi) .
$$

Then we have

$$
\begin{gathered}
b_{z}^{-1}-b_{\xi}^{-1}=-\left((A-z)(1+z A)\left(A^{2}+1\right)^{-1}(A-\xi)(A-z)^{-1} \eta_{2}(\xi), \eta_{1}(\bar{z})\right)+ \\
+\left((A-\xi)(1+\xi A)\left(A^{2}+1\right)^{-1} \eta_{2}(\xi),(A-\bar{z})(A-\bar{\xi})^{-1} \eta_{1}(\bar{z})\right)= \\
=-\left((A-\xi)(1+z A)\left(A^{2}+1\right)^{-1} \eta_{2}(\xi), \eta_{1}(\bar{z})\right)+\left((A-z)(1+\xi A)\left(A^{2}+1\right)^{-1} \eta_{2}(\xi), \eta_{1}(\bar{z})\right)= \\
=\left([(A-z)(1+\xi A)-(A-\xi)(1+z A)]\left(A^{2}+1\right)^{-1} \eta_{2}(\xi), \eta_{1}(\bar{z})\right)= \\
=\left(\left[A+\xi A^{2}-z-z \xi A-A-z A^{2}+\xi+\xi z A\right]\left(A^{2}+1\right)^{-1} \eta_{2}(\xi), \eta_{1}(\bar{z})\right)= \\
=\left((\xi-z) A^{2}+(\xi-z)\right)\left(\left(A^{2}+1\right)^{-1} \eta_{2}(\xi), \eta_{1}(\bar{z})\right)=(\xi-z)\left(\eta_{2}(\xi), \eta_{1}(\bar{z})\right) .
\end{gathered}
$$


By analogy with $(\tilde{A}-z)$, we can define $\left((\tilde{A})^{*}-\bar{z}\right)$ as perturbation of $\mathcal{H}_{-2}$-class.

Remark 9. Consideration of a singularly perturbed operator of $\mathcal{H}_{-2}$-class and its adjoint together also allows us to provide an additional description of both operators as a whole. Linear unbounded closed densely defined in $\mathcal{H}$ operator $\tilde{A} \neq A$ is singularly perturbed of $\mathcal{H}_{-2^{-}}$ class with respect to the operator $A$ (without loss of generality we assume $0 \in \rho(A) \cap \rho(\tilde{A})$ ), if both sets (15) are dense in $\mathcal{H}$ but herewith $\mathfrak{D} \subset \mathcal{H}_{+2}, \mathfrak{D}_{*} \subset \mathcal{H}_{+2}$.

Singularly perturbed $\mathcal{H}_{-2}$-class operator can also possess a new point $\lambda$ of the point spectrum $\sigma_{p}(\tilde{A})$, which was not in the spectrum $\sigma_{p}(A)$ of $A$.

Proposition 3. If the operator $\tilde{A} \in \mathcal{P}_{-2}^{1}(A)$ possesses a new eigenvalue $\lambda \in \mathbb{C}$, which was not by $A$, namely $\lambda \in \sigma_{p}(\tilde{A})$ but $\lambda \notin \sigma_{p}(A)$, then for correspondents eigenvectors $\varphi: \tilde{A} \varphi=\lambda \varphi$ and $\psi: \tilde{A}^{*} \psi=\bar{\lambda} \psi$, we have equalities:

$$
\begin{gathered}
(\lambda-z) b_{z}\left(\varphi, \eta_{1}(\bar{z})\right)=1, \quad \varphi=(A-z)(A-\lambda)^{-1} \eta_{2}(z), \\
(\bar{\lambda}-\bar{z}) \bar{b}_{z}\left(\psi, \eta_{2}(z)\right)=1, \quad \psi=\left(A^{*}-\bar{z}\right)\left(A^{*}-\bar{\lambda}\right)^{-1} \eta_{1}(\bar{z}),
\end{gathered}
$$

where $\eta_{i}(z)(i \in\{1,2\})$ denoted in Theorem $3, z \in \rho(A) \bigcap \rho(\tilde{A})$.

Proof. The proof is not essentially different from the proof of Proposition 1.

Theorem 4. For a given linear unbounded closed operator $A$ in a separable Hilbert space $\mathcal{H}$, a number $\lambda \in \mathbb{C}$ and a vector $\varphi, \psi \in \mathcal{H} \backslash \mathcal{H}_{+1}$ such that for some $z \in \rho(A), z \neq \lambda$ and $\left(\varphi,\left(A^{*}-\bar{\lambda}\right)\left(A^{*}-\bar{z}\right)^{-1} \psi\right) \neq 0$, there exists unique (up to the parameter) singularly non-symmetrically rank one perturbed operator $\tilde{A} \in \mathcal{P}_{-2}^{1}(A)$ such that $\lambda, \bar{\lambda}$ and $\varphi$, $\psi$ are its eigenvalues and eigenvectors, namely $\tilde{A} \varphi=\lambda \varphi$ and $\tilde{A}^{*} \psi=\bar{\lambda} \psi$. Herewith, the operator $\tilde{A}$ is defined by the resolvent (34) from Theorem 3: $\tilde{R}_{z}=R_{z}+b_{z}\left(\cdot, \eta_{1}(\bar{z})\right) \eta_{2}(z), \quad z \in \rho(A) \cap \rho(\tilde{A})$, with vector-valued functions $\eta_{1}(\bar{z})=\left(A^{*}-\bar{\lambda}\right)\left(A^{*}-\bar{z}\right)^{-1} \psi, \quad \eta_{2}(z)=(A-\lambda)(A-z)^{-1} \varphi$, and with the scalar-valued function $b_{z}^{-1}=(\lambda-z)\left(\varphi, \eta_{1}(\bar{z})\right),\left(\right.$ or $\left.\bar{b}_{z}^{-1}=(\bar{\lambda}-\bar{z})\left(\psi, \eta_{2}(z)\right)\right)$.

Proof. The proof has the same steps as the proof of Theorem 2, without taking into account technical details that require consideration of the $\mathcal{H}_{-2}$-class perturbations.

\section{4. $\mathcal{H}_{-2}$-class perturbations, that allow description by the $\mathcal{H}_{-1}$-class methods.}

Differences of coefficients $b_{z}$ in (3) and (31) are due to the fact that if, at least one of the vectors $\omega_{1}, \omega_{2}$ does not belong $\mathcal{H}_{-1}$, then the expression $\left\langle(\mathbf{A}-z)^{-1} \omega_{2}, \omega_{1}\right\rangle$ does not make sense at all.

Remark that if $\omega_{1}, \omega_{2} \in \mathcal{H}_{-1} \backslash \mathcal{H}$, then the denominator $b_{z}$ in $(31)$ can be written as follows

$$
\begin{aligned}
& \left((A-z)(1+z A)\left(A^{2}+1\right)^{-1} \eta_{2}(z), \eta_{1}(\bar{z})\right)+1 / \alpha+\tau= \\
= & \left\langle(\mathbf{A}-z)^{-1} \omega_{2}, \omega_{1}\right\rangle-\left\langle A\left(\mathbf{A}^{2}+1\right)^{-1} \omega_{2}, \omega_{1}\right\rangle+1 / \alpha+\tau,
\end{aligned}
$$

and we can put $\tau=\left\langle A\left(\mathbf{A}^{2}+1\right)^{-1} \omega_{2}, \omega_{1}\right\rangle$. Thus, Definition 1 becomes a partial case of Definition 2.

But if at least one of the vectors $\omega_{1}, \omega_{2}$ does not belong $\mathcal{H}_{-1}$ and herewith $\omega_{1} \neq \omega_{2}$, then it is possible that the expression $\left\langle(\mathbf{A}-z)^{-1} \omega_{2}, \omega_{1}\right\rangle$ has a sense. For example, it is easy to find two functions $f(x)$ and $g(x)$, both of which do not belong to $L_{2}(\mathbb{R}, d x)$, but the integral $\int_{-\infty}^{\infty} f(x) g(x) d x$ exists. The observed phenomenon is possible only with nonsymmetric perturbations (as opposed to symmetric one). 


\section{REFERENCES}

1. S. Albeverio, F. Gesztesy, R. Høegh-Krohn, H. Holden, Solvable models in quantum mechanics. Second edition, With an appendix by Pavel Exner, AMS Chelsea Publishing, Providence, RI, 2005, xiv +488 p.

2. S. Albeverio, R. Hryniv, L. Nizhnik, Inverse spectral problems for nonlocal Sturm-Liouville operators, Inverse Problems, 23 (2007), 523-535.

3. S. Albeverio, P. Kurasov, Singular perturbations of differential operators; solvable Schrödinger type operators, London Mathematical Society Lecture Note Series, V.271, Cambridge University Press, Cambridge, 2000, 265 p.

4. S. Albeverio, L. Nizhnik, Schrödinger operators with nonlocal point interactions, J. Math. Anal. Appl., 332 (2007), 884-895.

5. Y. M. Berezansky, J. Brasche, Generalized selfadjoint operators and their singular perturbations, Methods Funct. Anal. Topology, 8 (2002), №4, 1-14.

6. Yu.M. Berezansky, Z.G. Sheftel, G.F. Us, Functional Analysis, V.1,2, Birkhäuser Verlag, Basel-BostonBerlin, 1996, XIX +426 p.

7. M.E. Dudkin, T.I. Vdovenko, Dual pair of eigenvalues in rank one singular perturbations, Mat. Stud., 48 (2017), №2, 156-164.

8. M.E. Dudkin, T.I. Vdovenko, On extensions of linear functionals with applications to non-symmetrically singular perturbations, Methods Funct. Anal. Topology, 24 (2018), №3, 193-206.

9. T. Kato, Perturbation theory for linear operators, Die Grundlehren der mathematischen Wissenschaften, V.132, Springer-Verlag New York, Inc., New York, 1966, xix +592 p.

10. V. Koshmanenko, M. Dudkin, The method of rigged spaces in singular perturbation theory of selfadjoint operators, Operator Theory: Advances and Applications, V.253, Birkhäuser, Springer International Publishing, Switzerland, 2016, xii +237 p.

11. B.S. Mityagin, The spectrum of a harmonic oscillator operator perturbed $\delta$-interactions, Integr. Equ. Oper. Theory, 85 (2016), 451-495.

12. M.I. Vishik, On general boundary-value problems for elliptic differential equation, Trudy Moskow. Mat. Obshchestva, 1 (1952), 187-246.

National Technical University of Ukraine Igor Sikorsky Kyiv Polytechnic Institute

Kyiv, Ukraine

dudkin@imath.kiev.ua

oduzen@ukr.net

Received 08.04.2021

Revised 14.10.2021 